\title{
Flexibility and Rigidity of Cross-Linked Straight Fibrils under Axial Motion Constraints
}

\author{
Gyula Nagy KEM
}

Szent István University Ybl Miklós Faculty of Architecture and Civil Engineering Thököly út 74, HU 1146, Budapest, Hungary

E-mail: nagy.gyula@ybl.szie.hu

Abstract: The straight fibrils are stiff rod-like filaments and play a significant role in cellular processes as structural stability and intracellular transport. Introducing a 3D mechanical model for the motion of braced cylindrical fibrils under axial motion constraint; we provide some mechanism and a graph theoretical model for fibril structures and give the characterization of the flexibility and the rigidity of this bar-and-joint spatial framework. The connectedness and the circuit of the bracing graph characterize the flexibility of these structures. In this paper, we focus on the kinematical properties of hierarchical levels of fibrils and evaluate the number of the bracing elements for the rigidity and its computational complexity. The presented model is a good characterization of the frameworks of bio-fibrils such as microtubules, cellulose, which inspired this work.

Keywords: fibrils, cross-links, flexibility, rigidity, optimization, graph connectivity

\section{Introduction}

The fibril network literature declares that the cross-linked fibril structure is complicated and consist of redundant connections [34, 42, 70, 29, 5]. These redundancies are necessary the loading, the signal transporting, and the stability point of view. However, technical systems or the structures of the natural or social sciences have to be rigid and/or flexible some case at the same time [36, 11, 19, 30, 17, 15]. This paper characterizes the rigidity and mobility of rod-like fibrils in biological systems. The rod-like fibril structure with redundant bracing elements is safe if some of the bracing elements would collapse than the remainders make the structure rigid yet. Celluloses [24, 25, 63, 55, 10, 43, 66, 64], Fibrin [14], Collagens, Minerals, Microtubules, [76, 8, 18, 56, 39, 38, 41, 49, 77, 28, 1, 68], Chitins [58, 61, 62, 33] selfassemble into thick, hierarchically ordered, stiff fibers through electrostatic and hydrophobic interactions,. The network stiffness becomes surprisingly insensitive to network concentration, demonstrate how a simple model for networks of elastic fibres can quantitatively account for the mechanics of reconstituted collagen networks. We provide a discrete model that characterizes the flexibility and rigidity of braced framework of the fibrils. The model gives a good characterization in the case when the Young modulus is high enough, the order of magnitude not less than 1GPa for the fibers and the crosslinks also. The paper gives a model for further improvements. This model provides an input to other tests that take account of the exact mechanical properties of the fibrils, the cross-links, and the matrix. The mineral crystals in nano bone structure have a large aspect ratio. Hence, the direction of its displacement is the same as the macro fibrils that contain them and the micro fibril that connect them. Besides bone, other biological materials like shell, dentin, spider silk, wood, and chitin or the microstructural model of the axonal cytoskeleton [1] show similar structure despite their complex hierarchical structures. They are arranged in grid position in cross-section view, cross-linked by braces with multiplicity, which consist of similar material. The lower bound of the distance between any two cylinders in the packing is 
the thickness of the cylinder. In the case of dense packing of the cylinders (in Bundle or Layer structure), they can move only in its axial direction; the adjacent cylinders obstruct the lateral motions. Notwithstanding, if the axial motion constraints are not satisfied in a cross-linked fibril structure than our model gives a necessary condition for the rigidity of the bar-joint structure. If the SFF of the a cross-linked fibril structure is not rigid then its bar-joint structure is not rigid. Hence, further cross-links have to be applying for the rigidity that is count by the Maxwell's rank condition for the further method that should take account of the exact mechanical properties of the fibrils, the cross-links, and the matrix.

\subsection{Bar-and-Joint Framework}

The bar-joint framework: One of the simplest structures in statics is the bar-and-joint framework, that consists of optimal bars connected by rotatable joints, i.e. the bar lengths and the bar and joint incidences must be preserved.

\subsubsection{The rigidity of bar-and-joint framework}

Firstly, we give a definition of the rigidity of the bar-and-joint framework.

Definition 1: A framework is rigid if any continuous motion of the joints that keeps the length of every bar fixed, also keeps the distance fixed between every pair of joints.

It is a preservation of distances the joints during any continuous motion of the joints.

\subsubsection{The infinitesimal rigidity of bar-joint framework}

The infinitesimal motion is a special case of virtual motion; that refers to a virtual change in the position such that the constraints remain satisfied (possible motions).

Figure 1

The rigid body motions refer to the trivial infinitesimal motion. The Definition 1 allows frameworks that have infinitesimal motions. In the statics for the rigid structure, the non-trivial infinitesimal motions of the joints have not permitted [67, $54,60,57,51,59,65,40]$.

Definition 2: A framework is infinitesimally rigid if it only has trivial infinitesimal motions.

We can see the central joints of the framework on Figure 1. It has an infinitesimal motion up and down, perpendicularly to the plane of the structure. If a framework is infinitesimally rigid, we require first-order preservation of distances during the infinitesimal motions of all joints. We have to decide the rank of rigidity matrix of the framework.

Let $\left(\mathrm{x}_{\mathrm{i}}, \mathrm{y}_{\mathrm{i}}, \mathrm{z}_{\mathrm{i}}\right)$ be the coordinates of the joint Pi of a bar-joint structure, where $(1 \leq \mathrm{i} \leq$ $n$ ). A bar between the joints $P_{i}$ and $P_{j}$ determines the distance from $P_{i}$ to $P_{j}$, there for it is constant, by differentiating its square, leads to the next equation:

$$
\left(x_{i}-x_{j}\right)\left(\dot{x}_{i}-\dot{x}_{j}\right)+\left(y_{i}-y_{j}\right)\left(\dot{y}_{i}-\dot{y}_{j}\right)+\left(z_{i}-z_{j}\right)\left(\dot{z}_{i}-\dot{z}_{j}\right)=0
$$

Where, the velocity coordinates $\dot{\mathrm{x}}_{\mathrm{i}}, \dot{\mathrm{y}}_{\mathrm{i}}, \dot{\mathrm{Z}}_{\mathrm{i}}$ are the varieties. Hence, if we use bars between joints, and the number of bars is e, then we get a system with e pieces of equations. The matrix representation of the equation system is the next:

$$
A u=0
$$

Where $\mathrm{u}$ is the column vector of velocity, and $\mathrm{A}$ is an ex $3 \mathrm{n}$ rigidity matrix. In statics, rigidity does not even allow infinitesimal motions. In this case, the equation (2) has 
the trivial solution only (i.e. the rigid body like motions). The rigid body motion of the joint keeps fixed the distance between the pairs of the joint. If the joints of the framework have motions, that different from the rigid body motion, then the framework is not rigid. In this case the $\operatorname{rank}(A)<3 n-6$. The framework is rigid if and only if the $\operatorname{rank}(A)=3 n-6$, see: [47, 67, 60, 72, 50, 37, 54, 57]. Maxwell (1864) gave this characterization but with his result, the time complexity of deciding the rigidity is $O\left(e^{3}\right)$.

From this point, the infinitesimal motion and the infinitesimal rigidity refers to motion and rigidity respectively.

\subsubsection{Some bar-joint framework that has better rigidity characterization}

We want to characterize the rigidity in a simpler way. Such characterizing has known to square grids and annex building and many other structures.

It has shown that the rigidity of the structure depends on the places of the bracing elements rather than on their number. The geometric methods can be very efficient but are only applicable to those parts of the problems where the places of the joints are dependent. There are interesting results for lattices, tessellation, and other repetitive structures, see: $[6,3,60,50,20,21,59,51,27,32,7,48,65,46,57,12]$.

The mentioned results are better from an algorithmic point of view, because the size of the bracing graph of the framework, and hence the time complexity of the proposed algorithm, is smaller then consider the rank of the rigidity matrix.

\section{Bracing Straight Fibril}

\subsection{The Structure of Straight Fibril}

This paper gives a graph theoretical characterization of the rigidity of right circular cylinders with equal radius and different heights, where heights are significantly greater, then the radius. These cylinders can connect with each other by bracing elements, on their surfaces; these connections firstly substitute by bars, later we will use other type bracing elements also. The bracing element refers BE. The cylinders correspond to Straight Fibrils, referred to as Straight Fibrils, SF because they can move back and forth only; these are 1D segments, they can move on lines in 3D the consequence of the axial motion constraint. We take a sequence of Straight Fibrils that are in the axis of the cylinder, and the distance of the neighbouring joints, they connect the Straight Fibrils are equal to the distance between the neighbouring bracing elements along the axis of the cylinder. If the coaxial cylinders connect to each other with their full base faces, then the corresponding Straight Fibrils are attached. We can see four cylinders on the left in Figure 2 and the corresponding SF framework on the middle of the figure. If there are some bracing bars between the joints of two SFs, we refer to them as BEs or bars. We visualize our structures as 3D, effectively in our model, the joints are zero-dimensional, the braces and Straight Fibrils are 1D.

Figure 2

Having applied the rigidity theory for this unique bar-joint structure we give some necessary and sufficient condition for the rigidity of this structure that consists of straight fibrils and their movement under direction constraint. Combinatorial characterizing is not known to straight fibril at direction constraint in 3D space, however in (Recski 1989), frameworks were discussed, in which joints placed to horizontal or vertical tracks (i.e. they can move only in the prescribed direction) in 
the plane. In this paper, we characterize the motion of the fibrils in 3D under direction constraint, and the directions are arbitrary.

There is no buckling the consequence of the motion constraint. The lengths of the Straight Fibrils are arbitrary in SF. The SFs and the BEs assembled a framework. We will demonstrate that if there are enough BEs in the right positions, then the framework will be rigid.

Definition 3: The SF framework consists of SFs and some BEs that link them together.

We refer the SF framework as SFF.

\subsubsection{The motions under direction constraints}

Figure 3

$\mathrm{SF}$ as a rigid bar under direction constraints can only move in the same direction as its axis. We will use a bar as a BE between two SF-s, they are under direction constraint. The next theorem is analogous the equation (1) in the Maxwell approach.

Theorem 1: The BE is not perpendicular to neither of the two axes of the Straight Fibrils, if and only if both Straight Fibrils have infinitesimal motion (not zero), or none of the Straight Fibrils have any.

Proof: (by contradictions). If the SFs are parallel, then those infinitesimal motion components are equal. Otherwise, their projections to the axes of the cross-linked bar are different therefore the $\mathrm{BE}$ is not rigid.

If the SFs are not parallel, then their infinitesimal motion components are not equal. However, it is not possible that one of them is not zero, and the other is zero. In this case, their projection to the axis of the cross-linked bar are different, see Figure 3, one of them is not zero, and the other one is zero. Hence, the cross-linked bar could not be rigid.

Comment 1. If the BE is perpendicular to one of the SF-s, then the infinitesimal motions of the SF-s are independent of the other. In this case, the other has not any infinitesimal motion, if it is not perpendicular to the brace. If the BE perpendicular both of the Straight Fibrils, then they can move independently of each other. It is important that the bars do not connect to the Straight Fibrils at a right angle; in the opposite case, the brace refers to degenerate.

Comment 2. If the bracing bar is not perpendicular to any of their Straight Fibrils, then an infinitesimal motion of one of the Straight Fibril, generate the infinitesimal motion of the other Straight Fibril.

\subsubsection{The Bracing Graph}

Definition 5. $\mathrm{G}_{\mathrm{SFF}}\left(\mathrm{N}_{\mathrm{i}}, \mathrm{E}\right)$ is the bracing graph of SFF framework, where $\mathrm{N}_{\mathrm{i}}$, correspond to $\mathrm{SF}_{\mathrm{i}}$; and edge $\mathrm{N}_{\mathrm{i}}, \mathrm{N}_{\mathrm{j}}$ is an element of $\mathrm{E}$ if there is a non-degenerate $\mathrm{BE}$ between $\mathrm{SF}_{\mathrm{i}}$ and $\mathrm{SF}_{\mathrm{j}}$.

On the right side of Figure 2, we can see the bracing graph of the braced framework that we can see in the middle part of this figure. 


\subsection{The Flexibility in the Bundle.}

We assume that all of the SFs in the SFF are parallel. The SF can move to the direction of its axis, and the BEs restrict the displacements of the SFs.

Fit a coordinate system to the structure; that axis $\mathrm{Y}$ will be parallel to the SFs. If one of the bar end points has a motion or an infinitesimal motion in the Y-axis direction, then the other end point of this bar has the same motion or infinitesimal motion in the same direction due to the rigidity of the bars. There is not an $\mathrm{X}$ or $\mathrm{Z}$ motion component of the joint because the bars can only move in the $\mathrm{Y}$ direction, which is the consequence of the direction constraints condition.

Denote by $\mathrm{SF}_{\mathrm{i}}$; the element $\mathrm{i}$-th of the set of the SFs and denote by yi the infinitesimal motion of the $\mathrm{Y}$ coordinates of $\mathrm{SF}_{\mathrm{i}}$. Denote by $\mathrm{b}\left(\mathrm{SF}_{\mathrm{i}} ; \mathrm{SF}_{\mathrm{j}}\right)$ the bracing bar between Straight Fibrils $\mathrm{SF}_{\mathrm{i}}$ and $\mathrm{SF}_{\mathrm{j}}$. If there is a brace $\mathrm{b}\left(\mathrm{SF}_{\mathrm{i}} ; \mathrm{SF}_{\mathrm{j}}\right)$ than $\mathrm{y}_{\mathrm{i}}=\mathrm{y}_{\mathrm{j}}$. It is important that the brace has to be non-perpendicular to the Y-axis; in that case, it is possible that the infinitesimal motions of its endpoints are not equal in the direction Y; in this case, the bar is degenerate.

Figure 4

\subsubsection{The Rigidity of the SFF framework in Bundle}

The equations type (1) imply the Maxwell's stability criteria, analogously, the Theorem 1 imply the next theorem for parallel cross-linked fibrils in the 3dimensional space.

Theorem 2: The parallel Straight Fibril Framework in Bundle is rigid, if and only if its bracing graph $G_{S F F}\left(N_{i}, E\right)$ is connected.

Proof: If the bracing graph is connected then $y_{i}=y_{j}$ to each SF. Hence, they can move each other only into the direction constraint. Hence, they have the only motion of the translation that is a rigid body motion.

If the bracing graph is not connected then there exists an index $\mathrm{j}$ so that we cannot get from node $\mathrm{N}_{1}$ to node $\mathrm{N}_{\mathrm{i}}$ along edges in the bracing graph, hence the $\mathrm{y}_{1} \neq \mathrm{y}_{\mathrm{j}}$. Therefore, $\mathrm{SF}_{1}$ can move without reference to $\mathrm{SF}_{\mathrm{j}}$.

This theorem is analogous the next statement: a one-dimensional (on a line) bar-joint framework is rigid if and only if we can get from any joint to any other joint along by bars [50].

Comment 3. The motion or the infinitesimal motion of one of the Straight Fibril travels along not degenerated bars to the other Straight Fibrils they are in the same corresponding components of the bracing graph. The displacement of the Straight Fibrils does not change if they are connected to BEs.

Comment 4. Accordance with the Definition 2 the SFF in Bundle is rigid; apparently, all fibrils in the bundle could move in its matrix fluid independently the number of the BEs. The drag of the BEs obstructs this traffic, partially; and the BEs of the hierarchical structure of the bundle that is also fibril, resist this kind of motions.

Next, we consider two types of connection by BEs: in the first case, there are some bracing bars in the SFF, and the second case, snap bracing, we discuss the two instances in the different arrangement of SF. 


\section{SFs in Layers with different bracing connections}

Up to this point, we do not assume that the SF-s connect to the nearest neighbour, nor the regular arrangement of them, we also give up that the all of the SF are parallel in the framework, and we choose to connect nearest neighbours further. We suppose that axes of some SF are parallel, and its axes are in the same plane. They compose a layer.

\subsection{The rigidity of SF-s in one layer with regular BEs}

\subsubsection{The rigidity of SFs in one layer with regular BEs}

Firstly we consider the case when the SFs connect by regular BEs to the other SFs that would be in the same layer (first case), or will strictly be in another neighbouring layer (second case). In both cases, the BEs are not degenerate. We discussed the first instance since the second is special of its.

We suppose the directions of the fibrils that are in different layers are not parallel. Therefore, the SF can connect the all of the SF that are in the neighbouring layers. Hence, the average number of the nearest neighbour of one fibril will be more than the structure that was considered earlier, where the average number of the nearest neighbour is not more than six.

\subsubsection{The bracing graph of SFs in layer with regular BEs}

According to the earlier bracing graph definition $G_{S F F}(N i ; E)$ is the bracing graph our $\mathrm{SF}$ structure, where $\mathrm{Ni}$, correspond to $\mathrm{SFi}$; and edge $\mathrm{Ni}, \mathrm{Nj}$ is the element of $\mathrm{E}$ if there is non-degenerate BE between SFi; SFj. On Figure 5, we can see the bracing graph of the braced SFs in the layer.

Figure 5

Using Theorem 1, we could get to the next false theorem for SF framework in the layer structure.

False Theorem 3: The SF layer structure framework connecting by not degenerate bracing bars is rigid, if and only if, the bracing graph of them is connected. The simple connecting of the bracing graph implies the motions of all SF-s, or zero displacements of all SF-s, the consequence of Theorem 1. Take a pyramid for counter example. Let the four SF-s be on the four side edges. Connected them by four bracing bars, we experience that the symmetric cases are mechanism while the asymmetrical are rigid [54, 57, 35].

The other direction of the False Theorem 3 is also incorrect: If the SF layer structure framework connecting by not degenerate bracing bars is rigid, then the bracing graph of them is connected. See Theorem 4 and the right on below in Figure 5.

Therefore, we have to modify the definition of the bracing graph for a proper characterization of SF framework in a layer structure, and we have to change the prohibition of the degenerate BEs.

The rigidity of generic structure was considered in [2, 45, 60, 16, 37, 46, 35].

Definition 6: The BEs of the SF framework are generic if any not zero infinitesimal motion of any SF imply the not zero and not equal infinitesimal motion of any other not parallel SF along by BEs. 
Between the two parallel SFs, there are no generic BEs since each of the BEs imply the same displacement of the parallel SFs. Hence, the not zero infinitesimal motion of the one of the parallel SF imply not zero but same infinitesimal motion.

Definition 7: $G_{g e n}\left(N_{i} ; E\right)$ is the generic bracing graph of the SFF. The $\mathrm{N}_{\mathrm{i}}$ correspond to all of the $\mathrm{SF}_{\mathrm{i}}$, which are parallel and connected to each other along BEs; and edge $\mathrm{N}_{\mathrm{i}}, \mathrm{N}_{\mathrm{j}}$ belongs to $\mathrm{E}$ with multiplicity, if the BEs between $\mathrm{SF}_{\mathrm{i}}$ and $\mathrm{SF}_{\mathrm{j}}$, belong to the set of the generic BEs of SF framework.

If we complete the SFF some extra BEs between the neighbouring SF in the layers on Figure 5 , then the $G_{g e n}\left(N_{i} ; E\right)$ of the SFF will be a Graph with three nodes only. These correspond to each layer, one of them (it correspond to the middle layer) connect the other two by three-three edges. These corresponding cross-links are on the left-hand side picture of Figure 5.

Theorem 4: The SFF as layer structure framework connecting by generic bracing bars is rigid, if and only if all connected components of its bracing graph includes, at least, one circuit.

Proof: If the connected components of the bracing graph include, at least, one circuit then the corresponding SFs with generic braces are rigid. Along the elements of one of the cycles of the circuit is formed a rigid structure. If one of the fibril corresponding to a node of the cycle can move infinitesimally, than its neighbour also can move the consequence of the Definition 6. Along this cycle, we get back to the first node. The corresponding SFs can move, but the consequence of Definition 7 not equal to the earlier ones. There is itself between the previous one; this is contradicting. The SFs, which correspond to the nodes of the cycle, there are not any infinitesimal motion, they are infinitesimally rigid. Hence, the connected part of its component is also rigid the consequence of Theorem 1.

Converse also by contradiction i.e. the rigidity imply the connectivity and the existence of the circuit in the bracing graph: If one of the components of the bracing graph does not contain least one circuit, then the SFs corresponding to this component can move together the consequence of the Comment 2. Therefore, SFF is not rigid.

In the case of two fibrils crosslinked by two generic braces, the proof is visualized in front of the left-hand side picture on Figure 6 . A virtual displacement $\underline{F}_{1}$ of the upper $\mathrm{SF}$ imply displacement $\underline{\mathrm{B}}_{1}$ in the left-hand side BE. Hence, in the lower SF has displacement $\underline{F}_{2}$, its projection to the left-hand side $B E$ is also $\underline{B}_{1}$. The right-hand side joint of the lower fibril also has displacement $\underline{F}_{2}$ in consequence of the constant fibril lengths between two joints. The displacement of the right-hand side joint is $\underline{B}_{2}$. Hence, in the upper $\mathrm{SF}$ has displacement $\underline{\mathrm{F}}_{3}$, its projection to the right-hand side $\mathrm{BE}$ is also $\underline{B}_{2}$. The $\underline{\mathrm{F}}_{3} \neq \mathrm{F}_{1}$ in the case of generic BEs, this is contradicting, because they have to be equal the consequence of the constant fibril lengths between two joints. The SFF behind this framework is not generic.

Figure 6

Comment 5. If the bracing graph is circuit free therefore tree graph, hence we get from node $\mathrm{N}_{1}$ of the graph to the node $\mathrm{N}_{2}$ along connected edges. The infinitesimal motion of the corresponding Straight Fibril $\mathrm{SF}_{1}$ travels connecting with nondegenerated bars to the other Straight Fibrils of the corresponding nodes of the tree graph and get to $\mathrm{N}_{2}$. The infinitesimal motion of the Straight Fibril may change along the not degenerated BEs, but any of them is not zero. 


\subsection{The rigidity of SF-s with snap BEs}

\subsubsection{The rigidity of SF-s with snap bracing}

We consider the case when two SFs touch each other in a snap point. This touching point refers Snap; this fixed both fibrils if they are not parallel, and they are under the direction constraints. If they are parallel, then they can move together to them direction. In the bar-joint structure, On Figure 6, we can see a Snap. The BEs connect the joints of bars they have corresponded the touching fibrils. The length of these BEs is $2 r$, where $r$ is the radius of the cylinder type fibrils. We referred it a short brace. In the case of short brace the requirement of infinitesimal rigidity is not satisfied, the infinitesimal motion is allowed by the short brace to the direction of the axes of the bars. Hence, we have two possibilities, firstly correspond only one joint to the short brace and their two joints; in this case, we have to change the original geometry (the distance of the SF would be zero). Secondly, we have to apply two extra bars instead of the short brace, connected the joints of the short brace of one of the SF to another new joint of the other SF that different from the other joint of the Short brace, see the bottom of Figure 7 with dashed lines. We give similar situation if we use the short brace and an arbitrary brace that not connect to it. It is also enough if we use any two different braces between the two fibrils.

Figure 7

If two SFs are in different directions, and they have a mutual snap, then they are fixed to each other.

Connecting to each other by snap, the SFF is rigid if and only if any of them connected components contain least two SFs, which are not parallel.

Proof: If the directions of some of the SFs are parallel than they could move, into that direction, but least one SF connects least one of the others, and they are not parallel. Hence, these two snapped not parallel SFs fix the system the consequence of Theorem 4, namely these snapped two SF-s give the proper circuit the other snaps ensure the connectivity.

Comments 6. Only the isolated fibril can move in this structure.

On the right-hand side below in Figure 4, we can see a part of a bracing graph of an SFF by snaps that show the number of the snap elements is nearly half of the number of the formal bracing bar elements for the rigidity.

\subsubsection{The rigidity of SF-s in with mixed BEs}

Both types of BEs could connect two neighbouring SF in the SFF these structures refer SFF with mixed BEs.

Definition: $G_{m i x}\left(N_{i} ; E\right)$ is the bracing graph of SF-s in layer structure where are snaps connection and regular bracing bars connections mixed. $\mathrm{N}_{\mathrm{i}}$, correspond to $\mathrm{SF}_{\mathrm{i}}$ and edge $\mathrm{N}_{\mathrm{i}}, \mathrm{N}_{\mathrm{j}}$ belongs to $\mathrm{E}$ with multiplicity $\mathrm{m}$ if there are $\mathrm{m}$ pieces of a regular $\mathrm{BE}$ between $\mathrm{SF}_{\mathrm{i}}$ and $\mathrm{SF}_{\mathrm{j}}$, and edge $\mathrm{N}_{\mathrm{i}}, \mathrm{N}_{\mathrm{j}}$ belongs to $\mathrm{E}$ with multiplicity two if there is snap element between $\mathrm{SF}_{\mathrm{i}}$ and $\mathrm{SF}_{\mathrm{j}}$.

Theorem 6. The SFF with mixed BEs is rigid, if and only if, all of the connected components of its bracing graph include at least one circuit.

The proof is similar to the proof of Theorem 4.

Comment 7. This theorem characterizes the flexibility and the rigidity of SFs in layers with mixed BEs. 
The SFs in a layer structure can connect almost all of the SFs that are in the neighbouring layers if the angle of the SFs in the neighbouring layers is large enough. Hence, the average number of the nearest neighbour of one fibril in the layer structure will be more than the bundle structure where the average number of the nearest neighbour is not more than six. If an SF connects $n$ pieces of BEs, then the layer structure are more cohesive than the bundle structure. In the case of removing, one SF from the structures is imperceptible, but in the case of removing some assigned neighboring SFs from the bundle is lighter, because a significant part of the BEs connects the assigned neighboring SFs to each other, contrary to the layer structure. Hence, the SFs rather burst than slip out from the layer structure, seeing the Figure 8, similarly the pictures in [62]

Figure 8

In the case of $3 \times 3 \mathrm{SFF}$ square grid, the number of the BEs that connects them to the bundle structure is nearly $12 \mathrm{n}$; contrary to the layer structure, it is easy to approximate $24 \mathrm{n}$. Intrinsically the proportion of the fixing BEs is approximately twice in a favour of the layer structure.

\subsection{A generic structure}

On Figure 9, we can see an SFF structure if the BEs are not generic, i.e., for instance, $b$ parallel $c$ than the SF4 can move up or down independently of the others. In the case of generic bracing is out of the question, seeing Theorem 4. The dimension of possibilities when bar b is parallel to bar c is zero-dimensional. Hence, this case is irrelevant to the possibilities, when they are not parallel. Our structure is generic if the set of coordinates $\left(x_{i}, y_{i}, z_{i}, X_{j}, Y_{j}, Z_{j}: J_{i} \in J, S F_{j} \in S F F\right)$ are algebraically independent over $\mathrm{Q}$ (rational numbers). The $\mathrm{J}$ signs the set of the ball joint, that connects the BEs to the SFs, and $x_{i}, y_{i}, z_{i}$ is the coordinate of the ball joint $\mathrm{J}_{\mathrm{i}}[2,45$, $60,54,57,35]$. The $X_{j}, Y_{j}, Z_{j}$ there are the coordinates of one of the direction vector of Straight Fibril SF . This definition precludes the parallel SFs from the generic SFF. In the Definition 6 was sufficient to prevent the case of the bars that connect parallel SFs.

Figure 9

\subsection{The decision the rigidity of SFs}

Our result provides a very fast method for the decision of flexibility or the decision of rigidity of a given of SFF. This novel computational procedure in case of the SFF framework in Bundle based on the next procedure:

1. Given a fibril structure with BEs.

2. Build the bracing graph of the structure.

3. Is the bracing graph connected?

Hence, the decision of rigidity of SFF originates in the decision of graph connectivity. The complexity of the decision problem of the rigidity of SFF in the case of $n$ pieces of SF and m pieces of BE using Maxwell's result from 1864 is $O\left((m+n)^{3}\right)$ if we use Gaussian elimination for the rank of the rigidity matrix. For the adjacency list representation of the graph, the total time of the algorithm is $O(m+n)$ 
as its space is also. Accordingly the rigidity of the Straight Fibril is approximately a $(n+m)^{2}$ times faster than the simulations based on Maxwell' result, hence, the simulated numbers of fibril approximately increase from some hundred to some million.

\subsection{The safety of SFs}

In the literature, of real fibril declare that the network of the fibril consists of redundant connections. These redundancies are important the point of view the loading and the safety of the rigidity of the network. The fibril structure is a safety if some of the BEs collapse while the remainders have kept the rigid structure yet, i.e. its bracing graph satisfies the corresponding criteria i.e. the connected components include circuits. Similar problems are significant and well-studied optimization problems in graph theory, and network analysis. We introduce the case of the SFF in the bundle.

The connectivity augmentation problem is the next in our case: given a bracing graph and a positive integer $\mathrm{k}$, we find a minimum number of new edges in the bracing graph, that the results will be k-node-connected and/or k-edge-connected bracing graph. Hence, if we remove arbitrary $k-1$ nodes or edges the remained object not collapsed. The connectivity augmentation helps us to increase the safety of an already existing network by adding an optimal number of new connections for the rigidity of SFF.

It is an open question even if the graph $\mathrm{G}$ to be augmented is (k-1) vertex-connected. Polynomial algorithms have been developed only for $\mathrm{k}=2,3$, 4 by Eswaran [13], Watanabe [69] and Hsu [31], respectively. In network design, it is often of interest to know how sensitive a particular property of a network is to changes in the graph structure, like the removal or failure of edges. We focus on the edge-connectivity of a graph. The connectivity interdiction asks to decrease the edge-connectivity of a graph maximally by removing a limited set of edges. We can ask how many braces could be taken away at most before the graph disconnects. Hence, our framework would collapse. If the edge connectivity is $\mathrm{k}$ then we could take away $\mathrm{k}$ brace, the graph will become disconnected (not all of $\mathrm{k}$ brace are good for the disconnectivity), i.e. the framework will be a mechanism the consequence of the Comment 3. The connectivity interdiction is a reciprocal problem of edge-connectivity augmenting of a graph the weighted optimization problems have been studied in $[26,75]$.

\section{The boundary of the SFF flexibility and rigidity}

Our graph theoretical model can determine the flexibility or rigidity the structure of SFs. Maxwell's result counts the degree of freedom of the framework, and it solves the problem without the direction constraint also, our result for this case is not compatible.

It is expected that overall stiffness of a network consisting of rigid and soft elements be determined mainly by the elastic elements, which are the cross-linkers. In this case, the network stiffness would be insensitive to slight changes in the rigidity of the stiff elements that would be for instant the actin filaments. Structurally related hierarchical tissue systems exist throughout the human body, for example in partially mineralized tissues at tendon-to-bone attachments consisting of collagen cross-linked by stiff mineral particles $[22,44]$. Hence, the cross-links between fibrils are stiff, and the fibrils are viscoelastic, but analogous dissipative processes and randomness might be critical for optimal toughening $[53,40]$. This further suggests new principles for the design of synthetic fibril networks with collagen-like properties, as well as a mechanism for the control of the mechanics of such system. 
With a high resistance to bending and a stiffness of $2 \mathrm{GPa}$, the microtubules are undoubtedly the strongest cytoskeletal filaments in eukaryotic cells. As such, they play a unique role in some cellular processes, maintaining structural stability and providing a path for intracellular transport [9]. The microtubule structure is similar in all of the eukaryotic cells; it is harder justifiable that the assumption the motions of the fibril are under axial motion control.

Last, we mention, we can see a multi-layered arrangement of cellulose microfibril at the cell wall surface from onion scale, visualized the microfibril in the surface layer [76]. Note that microfibril merges into and out of regions of close contract. Hence, the direction constraint of the motion of the fibril it is not satisfied. The Artistic Image of the onion cell wall visualized from atomic force microscopy [76]. If we make the graph with one node on the fibril, and edges between the nodes in case adjacency of the fibril then this graph is not connected. Hence, the connected component of the fibril can move independently each other. In this case, the assumptions of the directed motions and parallel fibrils are not satisfied.

Our results provide strategies that maximize the number of the element of fragments and minimize preservation of intact cellulosic fibrils if the assumption is satisfied for instant in case of arboreal plants. To decrease lignin content while the skeleton of the plant is remaining rigid, we get higher sugar yield.

However, we hold, this model could be a good candidate for the future work in cell biology. Using the results of flexibility and rigidity of SFF, we can predict the motion of a structure of a million individual fibrils thanks to the reduction of the complex system.

\section{Conclusions}

We introduce a graph theoretical model for the flexibility and rigidity of the straightline fibril with bracing elements (BEs) structures under direction motion constraint was referred as Straight Fibril Framework (SFF).

The connectivity and the circuits of the bracing graph of the SFF characterize the flexibility and the rigidity of the structure using generic BEs in the most general cases.

We could estimate using this method how many BEs sufficient for the rigidity of the bounds of the fibril. Our results provide strategies that evaluate the number of the BEs of fragments and minimize preservation of intact fibrils.

The kinematical characterization taken provides a powerful model for exploring the mobility of these structures and gives a new approach to understanding the nanoscale behaviour of fibrils.

\section{Acknowledgement}

I thank András Recski and Judit Borsa for many productive discussions. This research was supported by the Hungarian National Science Foundation (grant number: 108947).

\section{Reference:}

1. Ahmadzadeh, H., Smith, D. H., Shenoy, V. B., 2015. Mechanical effects of dynamic binding between tau proteins on axonal microtubules during traumatic brain injury: predictions from a computational model. Biophys. J. 109, 2328-2337.

2. Asimow, L., Roth, B., 1978. The rigidity of graphs, Trans. Amer. Math. Soc. 245, 279-289.

3. Baglivo, J. A., Graver, J.E., 1983.Incidence and Symmetry in Design and Architecture. Cambridge University Press, Cambridge 
4. Bidone, T. C., Kim, T., Deriu, M.A., Morbiducci, U., Kamm, R. D., 2015. Multiscale impact of nucleotides and cations on the conformational equilibrium, elasticity and rheology of actin filaments and crosslinked networks. Biomech. Model. Mechanobiol. 14, 5, 1143-55.

5. Blundell JR, Terentjev EM. Forces and extensions in semiflexible

6. Bolker, E.D., Crapo, H., 1979. Bracing rectangular frameworks I. SIAM J. Appl. Math. 36, 3, 473-490.

7. Borcea, C.S., Streinu, I., 2010. Periodic frameworks and flexibility. Proc. R. Soc. A 466, 2633-2649.

8. Cheng. X., Pinsky, P. M., 2013. Mechanisms of self-organization for the collagen fibril lattice in the human cornea J R Soc Interface 10pp.

9. Conde, C., Cáceres. A., 2009. Microtubule assembly, organization and dynamics in axons and dendrites. Nat. Rev. Neurosci. 10, 319-332

10. Cosgrove DJ: Reconstructing our models of cellulose and primary cell wall assembly Current Opinion in Plant Biology 2014, 22:122-131

11. Csermely P. Weak Links: A Universal Key for Network Diversity and Stability. Heidelberg: Springer-Verlag, 2006.

12. Ellenbroek, W.G., Hagh, V.F., Kumar, A., Thorpe, M.F., van Hecke, M., 2015. Rigidity Loss in Disordered Systems: Three Scenarios. Phys. Rev. Lett. 114, 135501

13. Eswaran K. Tarjan R. 1976 Augmentation problems SIAM J. Computing5,4pp653665

14. Ferry, J. D., 1952. The mechanism of polymerization of fibrinogen. Proc Natl Acad Sci USA. 38. 7, 566-569.

15. Fletcher, D. A., Mullins, R. D., 2010. Cell mechanics and the cytoskeleton. Nature. $4637280485-492$.

16. Frank, A., 2011. Connections in combinatorial optimization, Oxford Lecture Series in Mathematics and its Applications, 38, Oxford University Press, Oxford.

17. Fratzl, P., 2008. Collagen: Structure and Mechanics Springer, New York.

18. Gardel, M. L., Shin, J. H., MacKintosh, F. C., Mahadevan, L., Matsudaira, P., Weitz, D. A., 2004. Elastic Behavior of Cross-Linked and Bundled Actin Networks. Science 304, 1301-1305.

19. Gáspár, M. E., Csermely, P. Rigidity and flexibility of biological Networks. Briefings in Functional Genomics. 11. 6. 443-456

20. Gáspár Zs., Radics N. Recski A. 1998Square Grids with Long Diagonals". Optimization Methods and Software 10pp217-231

21. Gáspár, Zs., Radics, N., Recski, A., 1999. Rigidity of square grids with holes. Computer Assisted Mechanics and Engineering Sciences. 6, 329-335.

22. Genin, G. M., 2014. Nanoscopic Injury with Macroscopic Consequences: Tau Proteins as Mediators of Diffuse Axonal Injury. Biophysical Journal. 106, 15511552

23. Genin, G. M., Kent, A., Thomopoulos. S., 2009. Functional grading of mineral and collagen in the attachment of tendon to bone. Biophys. J. 97:976-985.

24. Gibson LJ, Ashby MF, Harley BA: Cellular materials in nature and medicine Cambridge University Press. Cambridge; 2010

25. Gibson LJ: The hierarchical structure and mechanics of plant materials. Journal of the Royal Society Interface 2013, 9, 76: 2749-2766

26. Grandoni, F., Ravi, R., Singh, M., Zenklusen, R., 2014. New approaches to multiobjective optimization Math. Program. Ser. A146, 1-2, 525-554

27. Guest S.D., Hutchinson J.W. 2003On the determinacy of repetitive structures. Journal of the Mechanics and Physics of Solids 51pp383-391.

28. Gutjahr P, Lipowsky R, Kierfeld J. Persistence length of semiflexible polymers and bending rigidity renormalization. Europhys Lett 2006;76:994-1000.

29. Ha BY, Thirumalai D. Semiflexible chains under tension. JChemPhys 1997;106:4243-7. 
30. Head, D. A., Levine, A. J. \& MacKintosh, F. C. 2003. Deformation of cross-linked semiflexible polymer networks. Phys. Rev. Lett. 91, 108102

31. Hsu, T.-S., 2000. On four-connecting a triconnected graph J. Algorithms 35, .2, 202-234

32. Hutchinson R.G., Fleck N.A. 2006The structural performance of the periodic truss, Journal of the Mechanics and Physics of Solids, 2,25pp756-782

33. Ilnicka A. Lukaszewicz J. P.2015Discussion remarks on the role of wood and chitin constituents during carbonization Front. Mater.

http://www.frontiersin.org/Journal/Abstract.aspx?s=730\&name=carbonbased_materials\&ART_DOI=10.3389/fmats.2015.00020pp

34. Ingber DE. The architecture of life. Scientific Am 1998;278: 48-57.

35. Jackson, B., Owen, J.C., 2016. A characterisation of the generic rigidity of 2dimensional point-line frameworks. Journal of Combinatorial Theory, Series B. in press.

36. Jacobs, D. J., Rader, A.J., Kuhn, L. A., Thorpe, M.F., 2001. Protein flexibility predictions using graph theory. Proteins, 44: 150-165.

37. Jordán, T., Domokos, G., Tóth K., 2013. Geometric Sensitivity of Rigid Graphs. SIAM Journal on Discrete Mathematics. 27, 4, 1710-1726

38. Karsai, Á., Mártonfalvi, Zs., Nagy, A., Grama, L., Penke, B., Kellermayer, M.S.Z., 2006. Mechanical manipulation of Alzheimer's amyloid B1-42 fibrils. J. Struct Biol. 155, 316-326.

39. Kasza, K. E., Broedersz, C. P., Koenderink, G. H., Lin, Y. C., Messner, W., Millman, E. A., Nakamura, F., Stossel, T.P., MacKintosh, F.C., Weitz, D. A., 2010. Actin Filament Length Tunes Elasticity of Flexibly Cross-Linked Actin Networks. Biophysical Journal. 99, 4, 1091-1100.

40. Libonati, F., Nair, A. K., Buehler, M. J., 2013. Mechanics of collagenhydroxyapatite model nanocomposites. Mech. Res. Commun. 58, 17-23.

41. Licup, A.J., Muenster, S., Sharma, A., Sheinman, M., Jawerth, L.M., Fabry, B., Weitz, D.A., MacKintosh, F.C., 2015. Stress controls the mechanics of collagen networks. Proceedings of the National Academy of Sciences of the USA. 112. 31. 9573-9578

42. Liljenström H. Neural stability and flexibility: a computational approach. Neuropsychopharmacology 2003;28:S64-73.

43. Liu L, Shang-Guan K, Zhang B, Liu X, Yan M, Zhang L, Shi Y, Zhang M, Qian Q, Li J et al.: Brittle Culm1, a COBRA-like protein, functions in cellulose assembly through binding cellulose microfibrils. PLoS Genet 2013, 9:e1003704.

44. Liu, Y., Thomopoulos, S., Genin, G. M., 2014. Modeling the mechanics of partially mineralized collagen fibrils, fibers and tissue. J. R. Soc. Interface. 11, 20130835.

45. Lovász, L., Yemini, Y., 1982. On generic rigidity in the plane, SIAM J. Algebraic Discrete Methods 3, 91-98.

46. Malestein, J., Theran, L., 2013. Generic combinatorial rigidity of periodic frameworks. Advances in Mathematics. 233, 1, 291-331.

47. Maxwell, J.C., 1864. On the Calculation of the Equilibrium and Stiffness of Frames. Philos Mag. 27, 182, 294-299.

48. Mitschke, H., Schwerdtfeger, J., Schury, F., Stingl, M., Körner, C., Singer, R. F., Robins, V., Mecke, K., Schröder-Turk, G. E., 2011. Finding Auxetic Frameworks in Periodic Tessellations, Advanced Materials. 23, 22-23, 2669-2674.

49. Motte, S., Kaufman, L.J., 2013. Strain stiffening in collagen I networks. Biopolymers 99, 1, 35-46.

50. Nagy Gy. 1994 Diagonal bracing of special cube grids, Acta Technica Academiae Scientiarum Hungaricae, 106, 3-4, 256-273

51. Nagy Gy. 2001 Rigidity of an annex building. Structural and Multidisciplinary Optimization 22 1: 83-86

52. Nagy, G., Katona, J., 2010. Connectivity for Rigidity. Studies of the University of Zilina Mathematical series. 24, 1, 6, 59-64. 
53. Nair, A. K., A. Gautieri, M. J. Buehler. 2013. Molecular mechanics of mineralized collagen fibrils in bone. Nat. Commun. 4:1724.

54. Owen, J.C., Power, S. Frameworks symmetry and rigidity 2010 International Journal of Computational Geometry and Applications. 20, 6, p. 723-750

55. Park YB, Cosgrove DJ, A revised architecture of primary cell walls based on biomechanical changes induced by substrate-specific endoglucanases. Plant Physiol 2012, 158:1933-1943

56. Pelletier, O., Pokidysheva, E., Hirst, L. S., Bouxsein, N., Li, Y., Safinya, C. R., 2003. Structure of Actin Cross-Linked with $\alpha$-Actinin: A Network of Bundles. Phys. Rev. Lett. 91, 14pp

57. Power, S. C., 2014. Crystal Frameworks, Symmetry and Affinely Periodic Flexes, New York J. Math. 20, 1-29.

58. Prashanth, K.V.H., Tharanathan R.N. Chitin/chitosan: modifications and their unlimited application potential—an overview. Trends Food Sci Technol, 18 (2007), pp. $117-131$

59. Radics N. Recski A. 2002Applications of combinatorics to statics - rigidity of grids, Discrete Applied Math. 123pp473-485.

60. Recski A. 1989Matroid Theory and its Applications in Electric Network Theory and in Statics, Akadémiai Kiadó, Budapest and Springer, Berlin

61. Sachs, C., Fabritius, H., Raabe, D., 2006. Experimental investigation of the elasticplastic deformation behavior of mineralized cuticle by digital image correlation. J. Struct. Biol. 155, 409-425.

62. Sachs, C., Fabritius, H., Raabe, D., 2008. Influence of microstructure on deformation anisotropy of mineralized cuticle from the lobster Homarus americanus. J. Struct. Biol. 161, 2, 120-132.

63. Saitoh K. Ohno H. Matsuo S. 2013Structure and Mechanical Behavior of Cellulose Nanofiber and Micro-Fibrils by Molecular Dynamics Simulation Soft Nanoscience Letters3pp58-67

64. Svensson R.B. Hassenkam T. Hansen P. Magnusson S.P. 2010 Viscoelastic behavior of discrete human collagen fibrils J. Mech. Behav. Biomed. Mater., 3 (2010), pp. 112-115

65. Szymanski D. B.2014The kinematics and mechanics of leaf expansion: new pieces to the Arabidopsis puzzle Current Opinion in Plant Biology 22pp141-148

66. Tanaka H. Shibutani Y. Izumi S. Sakai S. 2012Planar mobility modes of eightbarjointed structures with a single degree of freedom. Int. J. Solids Struct. 49pp1712-1722.

67. Thomas L.H. Forsyth V.T. Sturcova A., Kennedy C.J., May R.P., Altaner C.M., Apperley D.C., Wess T.J., Jarvis M.C. 2013 Structure of cellulose microfibrils in primary cell walls from collenchyma Plant Physiol 161pp465-476

68. Thorpe, M., 1983. Continuous deformations in random networks. J. Non-Cryst. Solids 57, 355-370.

69. van den Bedem, H., Kuhl, E., 2015. Tau-ism: The Yin and Yang of Microtubule Sliding, Detachment, and Rupture. Biophysical Journal. 109, 11, 2215-2217.

70. Väkiparta, M; Yli-Urpo, A; Vallittu, PK 2004. Flexural properties of glass fiber reinforced composite with multiphase biopolymer matrix. Journal of Materials Science-Materials In Medicine. 15, 1, 7-11.

71. Wang. J. H.-C. 2006. Mechanobiology of tendon. Journal of Biomechanics 39. $1563-1582$

72. Watanabe T. Nakamura A. 1993A minimum 3-connectivity augmentation of a graph. J. Comput. Syst. Sci., 46,1pp 91-128

73. Whiteley, W., 1992. Matroids and rigid structures, in Matroid Applications ed. White, N., Encyclopedia of Mathematics and its applications. 40, 1-51.

74. Wood, G. C., Keech, M. K., 1960. The formation of fibrils from collagen solutions. 1. The effect of experimental conditions: Kinetic and electron-microscope studies. Biochem J. 75, 3, 588-598. 
75. Zenklusen, R., 2014. Connectivity interdiction. Operations Research Letters. 42, 67, 450-454.

76. Zhang T, Mahgsoudy-Louyeh S, Tittmann B, Cosgrove DJ: Visualization of the nanoscale pattern of recently-deposited cellulose microfibrils and matrix materials in never-dried primary walls of the onion epidermis. Cellulose 2014, 21:853-862.

77. Zimmermann, E. A., Ritchie, R. O., 2015. Bone as a Structural Material. Advanced healthcare materials. 4, 1287-1304.

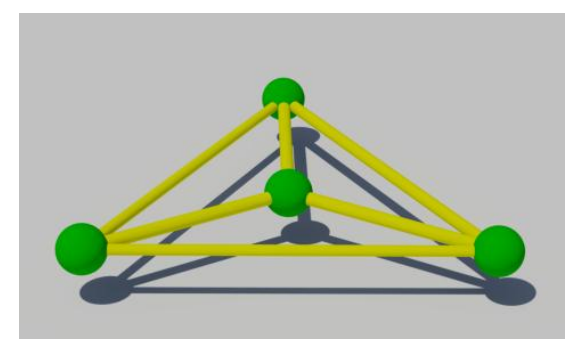

Figure 1

Infinitesimal motion: There are non-trivial infinitesimal motions of the central joints of the framework. The central points of its joints are in the same plane. The central joint can move infinitesimally into the normal direction of framework plane, at the beginning of this motion, no constraint restrict these motions.

The length of the shorter bars change in second-order, the preservation of all distances is first order.
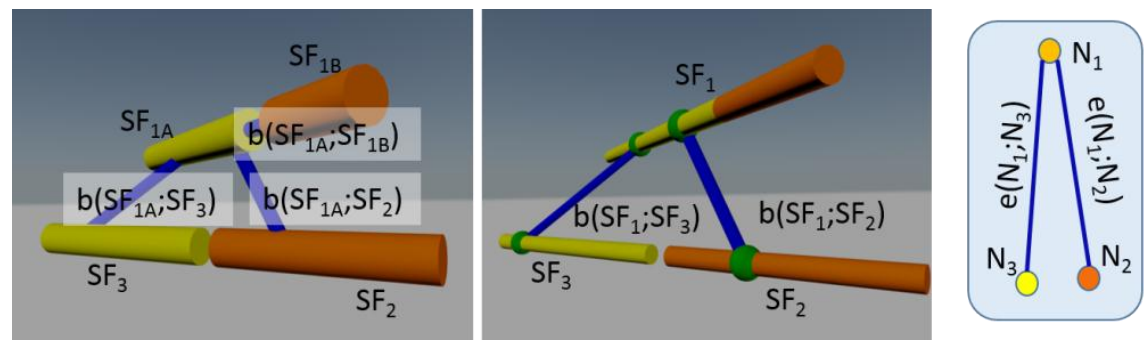

Figure 2

We illustrate the correspondences from fibrils structure to its graph across the SF framework. We can see four yellow cylinders on the left, the uppers connect with the base face with blue links, and the corresponding two Straight Fibrils are sticking together in the middle part of the figure. Two blue crosslinks are connecting both under cylinders with the upper left ones on the left part of the figure, the intersection of cylinders and cross-links have corresponded with ball joints. The bracing graph of the framework is to the right of the figure.

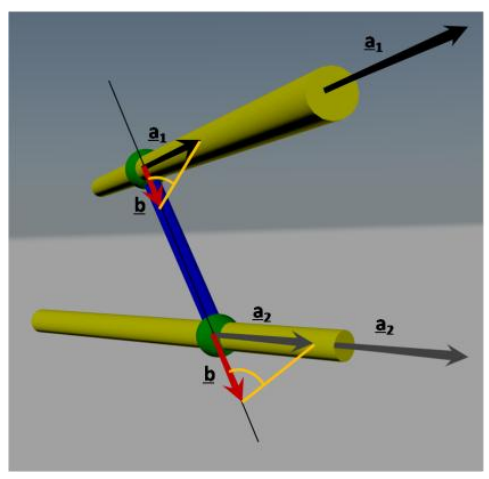


Figure 3

We can see two Straight Fibrils connected by a bar in skew position. The orthogonal projections of the motion vectors of the Straight Fibrils to the axis of the bar are equal the consequence of the rigidity of the bar.
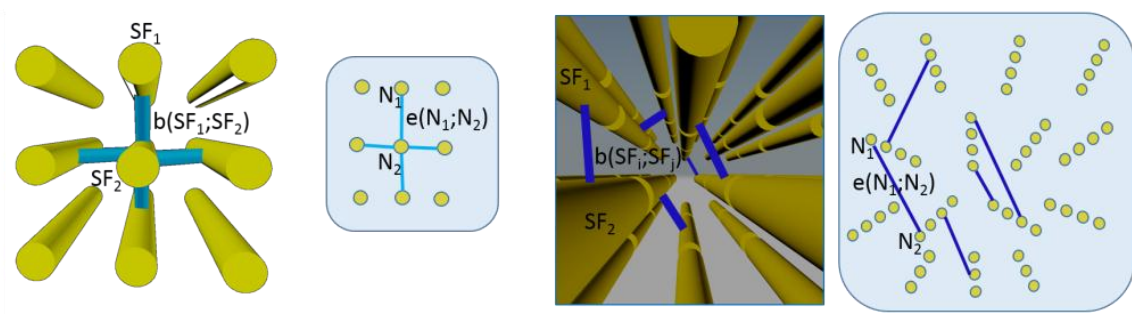

Figure 4

Two type of SFF in Bundle is in the figure the BEs connect the nearest neighbors. On the left part of the figure, we can see the SF-s in square grid positions and triangular grid position on the right. The bracing graph of the frameworks is on the right side of the cartoon of the braced framework. If we project the

Straight Fibrils of the framework and their BEs to the $\mathrm{Y}$ direction, then we get a structure that is isomorphic to the bracing graph. In the arrangement of the triangular grid, the Straight Fibrils have not braced links they are on the same axis. They do not compose a long Straight Fibril. Hence, each SF has a node in the graph on the right side.
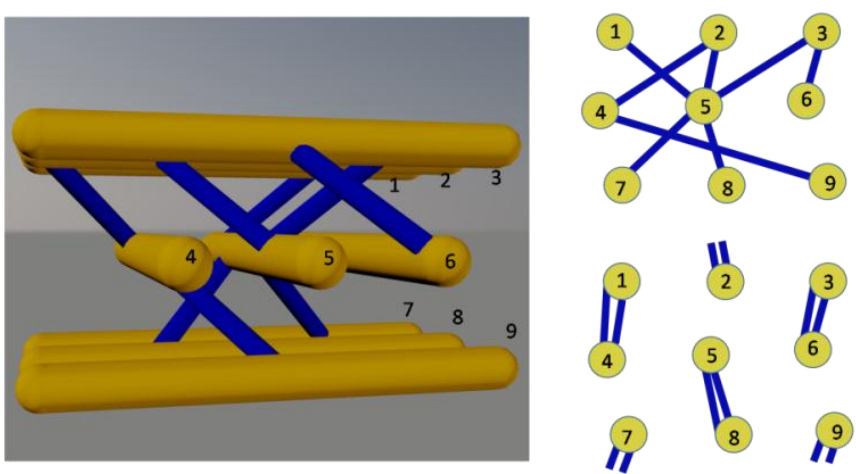

Figure 5

An SFF is arranged into layers. On the left, we can see an SF framework in layer structure; the joints are not signed. We can see on the right above the bracing graph $G_{S F F}\left(N_{i} ; E\right)$ of the SF framework on the left side. A part of the bracing graph of a potentially braced framework by snaps is shown on the right below.

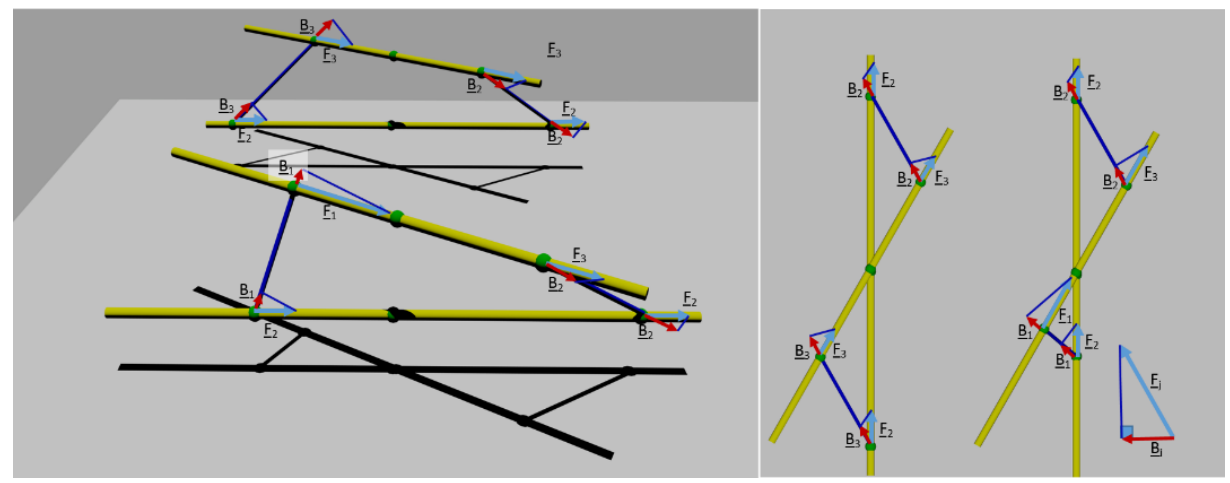

Figure 6

We can see a generic SSF and behind it a not generic SFF on the left picture of the figure. We can see on the right picture the top view of the two frameworks projected to a plane that parallels the both fibril. The 
under bar is symmetrical to the upper in the case of the left framework on this picture. In this case is no contradicting. In the right under part of the picture, we can see the projection of the displacement of the fibril to the connected bar.

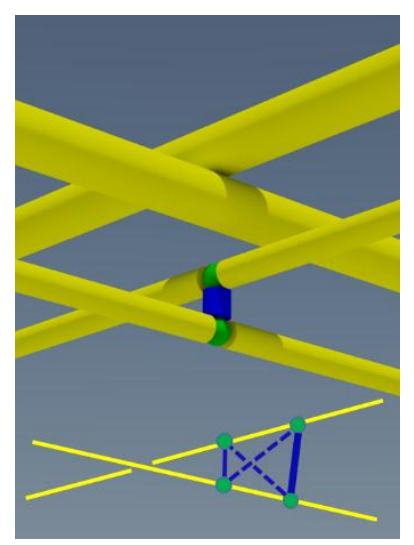

Figure 7

We can see on top of figure two not parallel SF with a snap. They cannot move to the directions of the axes of the fibrils. In the middle part of the picture, a possible bar-joint framework is shown that not suitable about the infinitesimal motions. Bellow, we can see a two possible SF framework that available one of with the dashed lines bars and the other with the solid lines bars.

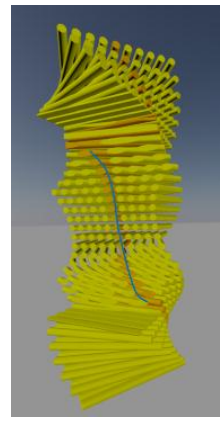

Figure 8

We can see the microstructure of fibrils in layer arrangements. Schematic depiction of the layer structure of the fibrils (seeing the curves) gives similar arrangement in hard tissues [61, 62]. Cross fracture of fibril structure mineralized cuticle from the lobster Homarus americanus shows an $180^{\circ}$ rotation of "vertical" fiber layers around the normal direction.
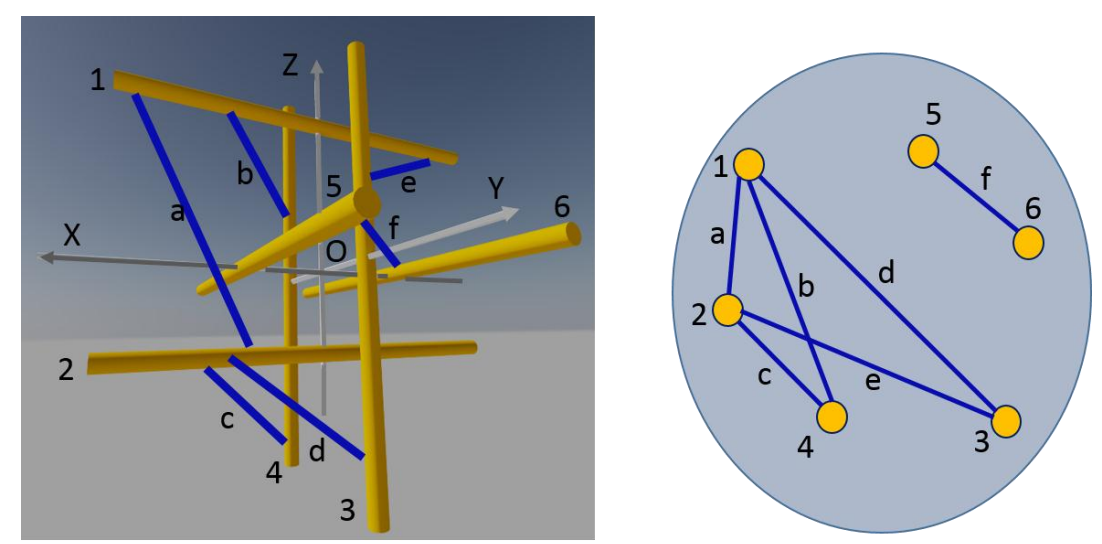

Figure 9

We can see a generic structure of SFF. On the right of the figure, we can see six fibrils signing by numbers; they are almost parallel to the coordinate axes in a couple; they can only move in the directions 
of the corresponding self-axis without BEs. Using the alphabetic signing BEs will they be flexible or not? We can see on the left its bracing graph. It has a component $(G(5,6 ; f))$ that not includes a circuit. The Straight Fibrils 5 and 6 , with bar $f$, can move together into them self-axis they show in the proximal direction of the axis $Y$; the others fix each other with them BEs. 